\title{
Sleep disorders in cerebellar ataxias
}

\author{
José L. Pedroso, Pedro Braga-Neto, André C. Felício, \\ Camila C.H. Aquino, Lucila B. Fernandes do Prado, \\ Gilmar Fernandes do Prado, Orlando G.P. Barsottini
}

\begin{abstract}
Cerebellar ataxias comprise a wide range of etiologies leading to central nervous system-related motor and non-motor symptoms. Recently, a large body of evidence has demonstrated a high frequency of non-motor manifestations in cerebellar ataxias, specially in autosomal dominant spinocerebellar ataxias (SCA). Among these nonmotor dysfunctions, sleep disorders have been recognized, although still under or even misdiagnosed. In this review, we highlight the main sleep disorders related to cerebellar ataxias focusing on REM sleep behavior disorder (RBD), restless legs syndrome (RLS), periodic limb movement in sleep (PLMS), excessive daytime sleepiness (EDS), insomnia and sleep apnea.
\end{abstract}

Key words: sleep disorders, ataxias, diagnosis.

\section{Distúrbios do sono nas ataxias cerebelares}

\section{RESUMO}

As ataxias cerebelares se caracterizam por uma enorme variedade de etiologias, cursando tanto com sintomas motores como também com sintomas não motores. Recentemente, várias evidências têm demonstrado uma frequência elevada de sintomas não motores nas ataxias cerebelares, especialmente nas ataxias espinocerebelares autossômicas dominantes (SCA). Dentre os sintomas não motores, estão os distúrbios do sono, que muitas vezes são sub-diagnosticados ou pouco valorizados. Nessa revisão, enfatizamos os principais distúrbios do sono relatados nas ataxias cerebelares, como transtorno comportamental do sono REM, síndrome das pernas inquietas, movimentos periódicos das pernas no sono, sonolência diurna excessiva, insônia e apnéia do sono.

Palavras-chave: distúrbios do sono, ataxias, diagnóstico.

Cerebellar ataxias comprise a wide range of neurodegenerative, congenital, mitochondrial, and metabolic diseases leading to central nervous system-related motor and non-motor dysfunctions ${ }^{1}$. Among these non-motor dysfunctions, sleep disorders have been recognized, although still under or even misdiagnosed. Their pathogenesis may be secondary to direct structural alterations of the sleepwake generating cells and networks or be the consequence of several indirect mechanisms ${ }^{1}$. Recently, a large body of evidence have demonstrated that non-motor manifestations are much more frequent than previously thought not only in autosomal dominant spinocerebellar ataxias (SCA), but also in several other neurodegenerative diseases such as synucleinopathies (Parkinson's disease, Lewy body dementia and multiple system atrophy), taupathies (Alzheimer's disease, frontotemporal dementia, corticobasal degeneration, progressive supranuclear palsy), amyotrophic lateral sclerosis, and prion diseases ${ }^{1}$.

The most frequent recognized sleep disorders in patients with neurodegenerative diseases are insomnia, hypersomnia, parasomnias, excessive nocturnal motor activity, circadian sleep-wake rhythm disturbances, respiratory dysrhythmias and sleep apnea ${ }^{1}$. It is crucial to recognize 
sleep disorders in neurodegenerative diseases not only to make an earlier diagnosis, but also to improve the quality of life ${ }^{1,2}$. In this review, we highlight the main sleep disorders related to cerebellar ataxias focusing on REM sleep behavior disorder (RBD), restless legs syndrome (RLS), periodic leg movement in sleep (PLMS), excessive daytime sleepiness (EDS), insomnia and sleep apnea ${ }^{1,3}$.

\section{Sleep disorders in autossomal dominant SCAs}

SCAs have been defined as a group of autosomal dominant ataxic disorders caused by degeneration of the cerebellum and its afferent and efferent connections. The majority of SCA are also complicated by the presence of additional neurological signs and non-motor symptoms, such as sleep disorders ${ }^{4}$. Additionally, extra-cerebellar alterations vary between genotypes ${ }^{4}$.

Sleep complaints, particularly RLS and PLMS, have been observed in some forms of SCAs, with a higher frequency in SCA1, SCA2, SCA3 or Machado Joseph disease (SCA3/MJD), and SCA6 $6^{1,5,6}$. Available evidence shows that the frequency of RLS in SCAs is significantly greater than in the general population ranging from 20 to $30 \%{ }^{6}$. In line with this we describe in the following section the most frequent sleep disorders seen mainly in SCAs 1,2,3 and 6.

Spinocerebellar ataxia type 1 (SCA1): RLS has been poorly reported in patients with SCA 1 . Among the few data available the frequency of RLS in patients with SCA1 is estimated to be $23 \%$ in a small sample ${ }^{6}$. Another study showed a similar frequency, but with even fewer patients. One study pointed out to a lack of association between RLS and periodic limb movement on polysomnography ${ }^{5}$. Interestingly, one study did not show evidence for RLS in SCA1 showing that the frequency actually varies according to patient selection or study methodology $y^{5,6}$. Recently, EDS and fatigue were also reported in two individuals with SCA1, suggesting that EDS in SCA1 may be an inherent consequence of the neurodegenerative disorder itself, and wake-promoting agents may be of benefit?

Spinocerebellar ataxia type 2 (SCA2): RLS has been described in patients with SCA2, and frequency ranges between 18 and 27\%5,6. Moreover, two previous studies have shown RBD in SCA2 patients characterized with REM without atonia and mioclonic jerks ${ }^{8,9}$. PLMS had a higher frequency in just one of the studies 9 .

Spinocerebellar ataxia type 3 or Machado Joseph disease (SCA3/MJD): SCA3/MJD is the most common autosomal dominant-subtype of ataxia worldwide ${ }^{4,10}$. The disease comprises a wide range of clinical manifestations, which include cerebellar ataxia, parkinsonism, dystonia, peripheral neuropathy, pseudoexophthalmos, lower motor neuron disease, with fasciculations and amyot-
Table 1. Non-motor symptoms of SCA3/MJD patients.

- Sleep disorders

- Psychiatric disorders (depression, anxiety and psychotic symptoms)

- Cognitive dysfunction

- Olfactory dysfunction

- Other non-motor symptoms: cramps, chronic pain and fatigue

rophy ${ }^{10}$. Recent studies also point out for a more diffuse neurodegeneration, which better explains the non-motor symptoms of the disease and possibly under-recognized, such as sleep disorders, memory deficits and executive impairment, olfactory dysfunction and psychiatry disturbances (Table 1$)^{11}$. In addition, the cerebellum itself has also been involved in non-motor functions. Recent clinical data has demonstrated high incidence of sleep complaints in SCA3/MJD ${ }^{12}$. Likewise, sleep complaints have been considered as an important modifier of health-related quality of life in SCAs ${ }^{12}$. The most frequent reported sleep complaints in SCA3/MJD are RLS ${ }^{13}$, $\mathrm{RBD}^{14}$, EDS ${ }^{14}$, sleep apnea ${ }^{12}$, and insomnia ${ }^{12}$. Curiously, not only these sleep disorders might be a frequent nonmotor symptom in SCA3/MJD, but may also precede the onset of cerebellar symptoms ${ }^{2}$.

When we consider SCA3/MJD patients, frequency rates of RLS are high, reaching about 55\% in several studies $^{12,14,15}$. Regarding the dopaminergic system dysfunction of primary RLS, it is speculated that RLS could reflect basal ganglia impairment in patients with $\mathrm{SCAs}^{5}$. Also, RBD has a higher frequency in SCA3/MJD patients when compared to the general population, reaching up to $50 \%{ }^{12}$. The underlying involvement of midbrain cholinergic and pontine noradrenergic systems seen in SCA3/ MJD patients could in part explain these higher rates of $\mathrm{RBD}^{2,3}$. Likewise synucleinopathies, RBD and RLS may precede any other clinical manifestation of cerebellar symptons by more than 10 years ${ }^{2}$.

High frequencies of EDS have been documented in SCA3/MJD patients ${ }^{12,14}$. Furthermore, in some series, insomnia was the most frequently reported sleep disorder $^{12}$. There is also a higher frequency of sleep apnea in SCA3/MJD than in general population ranging from 20 to $25 \%{ }^{12}$. Patients who reported snoring and possible apnea during sleep had longer disease duration and were older $^{12}$. Moreover, hypnagogic hallucinations are more related in SCA3/MJD patients than healthy subjects ${ }^{12}$. Figure describes some polysomnographic features in a patient with SCA3/MJD.

Spinocerebellar ataxia type 6 (SCA6): One study found a marked increase in PLMS indices in five patients with SCA6. Interestingly, all patients had a normal nerve 
conduction study excluding peripheral neuropathy as a cause of their limb complaints. The same study showed RLS in two of 5 patients and no RBD ${ }^{16}$. A larger study with 21 SCA6 patients found only one patient with RLS and sleep apnea ${ }^{13}$.

Table 2 is a brief summary of many studies concerning sleep disorders found in different SCAs subtypes. We have not found enough data to inform the frequency of sleep disorders in the SCAs other than SCA 1, 2, 3 and 6 , although some sporadic reports are available in the literature.

\section{Sleep disorders in recessive ataxias}

Recessive ataxias may be divided into five groups: Congenital, mitochondrial, metabolic, degenerative, and related to defects in DNA repair ${ }^{17}$. A limited number of data is available concerning sleep disorders in recessive ataxias.

Among congenital ataxias, Joubert syndrome and related disorders (JSRD) are the most remarkable. This term is adopted to describe a group of diseases that presents the "molar tooth sign" in brain imaging. JSRD is typically characterized by hypotonia, ataxia, respiratory disturbances, and abnormal ocular movements. The typical respiratory abnormalities are represented by episodes of apnea and/or hyperventilation that can range since from brief periods of hyperpnea until long attacks of apnea ${ }^{18}$. Although first described in awake state, the respiratory abnormality can occur during sleep. It has been described a sleep disordered breathing manifesting as repetitive tachypnea followed by central apnea, and isolated tachypnea during asleep, interestingly detected only in non-REM sleep ${ }^{19}$.

Friedreich ataxia (FA) is the commonest recessive ataxia worldwide, manifesting as progressive sensory and cerebellar ataxia, arreflexia, Babinski sign, often associated with cardiomyopathy, scoliosis and pes cavus ${ }^{17}$. Data on sleep disorders in patients with FA is poor. It

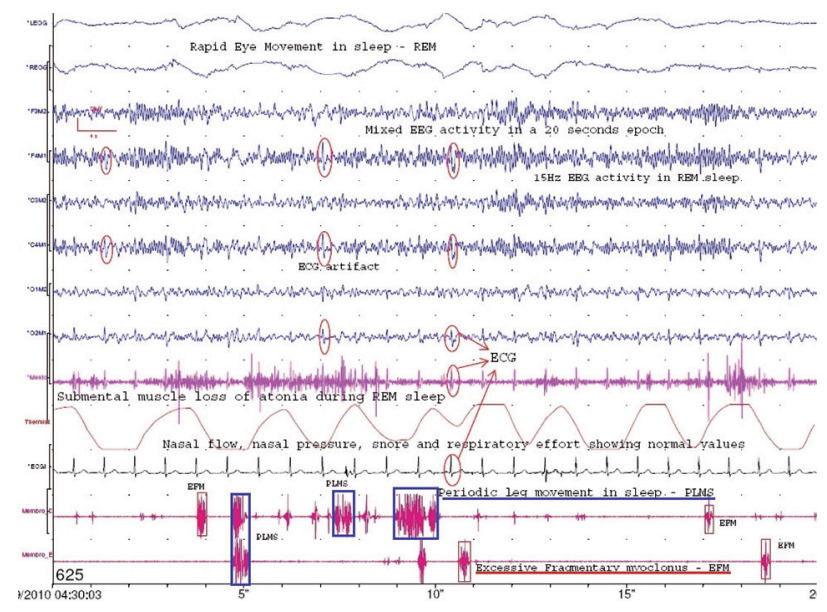

Figure. PSG of a 52 year-old-woman, with clinical and molecular diagnosis of SCA3/MJD. This is an epoch of 20 seconds of REM sleep showing its typical EEG features and a paradoxically high amplitude of the submental muscle, characterizing the phenomenon RWA for this interval. Note also PLMS and FM disclosed in both legs. PSG: polysomnography; EEG: electroencephalography; N3: stage 3 of non-REM sleep accounting for the old stage 3 and stage 4 of Rechtchaffen and Kales sleep scoring system; RWA: REM without atonia; FM: fragmentary myoclonus; PLMS: periodic leg movement in sleep.

has been published a case of mixed apnea, with obstructive and central apnea in one patient with late stage $\mathrm{FA}^{20}$.

A polysomnographic study in twelve patients with ataxia telangiectasia, a recessive disease that causes not only ataxia, but also cutaneos telangiectases, immunodeficiency, sino-pulmonary infections and lymphoreticular malignancies, was conducted to evaluate the patterns of sleep and respiration during sleep. The authors found decreased sleep efficiency when compared to normal values, eight patients with obstructive apnea and hypopnea, and four with central apnea, approximately half of them with oxygen desaturations ${ }^{21}$.

Despite the lack of enough previous studies concerning sleep disorders in recessive ataxias, we realized that the

Table 2. Frequency of sleep disorders in patients with various types of autosomal dominant cerebellar ataxias.

\begin{tabular}{lcccc}
\hline Sleep disorder & SCA 1 & SCA 2 & SCA 3/MJD & SCA 6 \\
\hline RLS & $23 \%(3 / 13)$ & $18 \%(2 / 11)$ & $56.7 \%(21 / 37)$ & $23.8 \%(5 / 21)$ \\
RBD & NA & $80 \%(4 / 5)$ & $45.9 \%(17 / 37)$ & NA \\
EDS & NA & NA & $45.3 \%(24 / 53)$ & NA \\
Insomnia & $50 \%(3 / 6)$ & $18 \%(2 / 11)$ & $37.7 \%(20 / 53)$ & $23.8 \%(5 / 21)$ \\
Nightmares & NA & NA & NA & NA \\
OSA & NA & NA & $22.6 \%(12 / 53)$ & NA \\
PLMS & NA & NA & NA & NA \\
Hypnagogic hallucinations & NA & NA & $38.4 \%(20 / 53)$ & NA \\
\hline
\end{tabular}

SCAs: spinocerebellar ataxias; RLS: restless legs syndrome; RBD: REM sleep behavior disorder; EDS: excessive daytime sleepiness; OSA: obstructive sleep apnea; PLMS: periodic leg movement in sleep; NA: not available. Statistical analysis of the table is reported in references 6, 8, 9, and not published information of our division of cerebellar ataxias. 
main sleep complaints are related to breathing. Therefore, physicians should be aware about sleep-breathing disorders in recessive ataxias and polysomnography study should be promptly performed in this situation.

\section{Sleep disorders in sporadic ataxias}

Multiple system atrophy (MSA) is a sporadic neurodegenerative disease characterized clinically by various combinations of cerebellar, parkinsonism, autonomic and pyramidal symptoms or signs. Pathologically is characterized by cell loss, gliosis and glial cytoplasmatic inclusions in several brain and spinal cords structures ${ }^{22}$. When cerebellar ataxia is the dominant clinical feature, the disease is termed MSA-C. The signs of cerebellar dysfunction include disorders of extraocular movements, ataxia of speech, ataxia of limbs movements and gait ${ }^{22}$.

MSA patients have multiple sleep-related disorder symptoms including EDS, insomnia, arrhythmic respiration, sleep apnea, $\mathrm{RBD}$, and sleep-related stridor with vocal cord paresis ${ }^{23}$. OSA and central sleep apnea frequently occur in MSA and may cause sudden death. RBD is closely related to neurodegenerative disorders, specially the synucleinopathies, and may precede motor symptoms of PD or MSA by years ${ }^{24}$. It has been demonstrated that REM sleep behavior disorder may precede other aspects of synucleinopathies by up to 25 years ${ }^{25}$. Another data comparing sleep disturbances in patients with PD, MSA and PSP demonstrated that patients with MSA showed higher risk of OSA and less frequent RLS ${ }^{26}$. The PRIAMO study, which analyzed non-motor symptoms in atypical and secondary parkinsonism, found that sleep disturbances were also common with a prevalence of approximately $70 \%$ in all diagnostic groups, including MSA patients ${ }^{27}$.

RBD and nocturnal stridor are considered red flags and may be the first symptoms of the disease ${ }^{22}$. Sleepdisordered breathing manifests as [1] central hypoventilation that reflects impaired automatic control of ventilation secondary to degeneration of the pontomedullary respiratory centers; and, more commonly, as [2] stridor and obstructive sleep apnea due to larynx narrowing secondary to combined vocal cord abductor paralysis and excessive adductor activation during inspiration ${ }^{23}$.

The recent identification of fragile X-associated tremor ataxia syndrome (FXTAS) associated with premutations in the FMR1 gene and the possibility of clinical overlap with multiple system atrophy has raised important questions. These neurodegenerative diseases may have similar clinical presentations, including sleep disorders ${ }^{28}$.

Alexander disease (AD) in its typical form is an infantile lethal leucodystrophy, characterized pathologically by Rosenthal fibers accumulation. Detailed clinical and genetic data from one study of 11 cases of adult-onset $\mathrm{AD}$ showed that the most frequent symptoms were related to bulbar dysfunction with dysarthria, dysphagia, dysphonia, pyramidal involvement and cerebellar ataxia. Sleep disorders were also observed in four cases ${ }^{29}$.

Sporadic fatal insomnia is a rare prion disease that has recently been recognized and is characterized by loss of sleep, oneiric stupor with autonomic/motor hyperactivity and somato-motor abnormalities, including pyramidal signs, myoclonus, dysarthria, dysphagia and ataxia ${ }^{30}$. In the new variant of Creutzfeldt-Jakob disease some patients demonstrated a combination of psychiatric symptoms, ataxia and severe sleep disorders ${ }^{31}$.

Although still unrecognized sleep disorders in cerebellar ataxias have been a recent issue of interest, and growing evidence has proving the importance of their identification. Not only because sleep disorders in cerebellar ataxias may precede the onset of motor symptoms, but also due to its impact in the health-related quality of life.

A similar problem found in the studies aforementioned is that the numbers of patients already evaluated are too small to conclude specific patterns of sleep disorders in a single group of cerebellar ataxias, for instance SCA3, and also limited information on the exact frequency of these sleep-complaints limits a full data interpretation. Therefore, future studies addressing sleep disorders in a large sample population with cerebellar ataxias are welcome. Additionally, a standardized assessment of these patients should be considered to allow data comparisons within different research centers.

\section{REFERENCES}

1. Raggi A, Ferri R. Sleep disorders in neurodegenerative diseases. Eur Neurol 2010;17:1326-1338.

2. D'Abreu A, Friedman J, Coskun J. Non-movement disorder heralds symptoms of Machado Joseph disease years before ataxia. Mov Disord 2005; 20:739-741

3. Frenette E. REM sleep behavior disorder. Med Clin North Am 2010;94: 593-614.

4. Schols L, Bauer P, Schmidt T, Schulte T, Riess O. Autosomal dominant cerebellar ataxias: clinical features, genetics, and pathogenesis. Lancet Neurol 2004;3:291-294.

5. Reimold M, Globas C, Gleichmann M, et al. Spinocerebellar ataxia type 1,2 and 3 and restless legs syndrome: striatal dopamine D2 receptor status investigated by [11C]raclopride positron emission tomography. Mov Disord 2006;21:1667-1673.

6. Abele M, Bürk K, Laccone F, Dichgans J, Klockgether T. Restless legs syndrome in spinocerebellar ataxia types 1, 2, and 3. J Neurol 2001;248: 311-314

7. Dang D, Cunnington D. Excessive daytime somnolence in spinocerebellar ataxia type 1. J Neurol Sci 2010;290:146-147.

8. Boesch SM, Frauscher B, Brandauer E, Wenning GK, Högl B, Poewe W. Disturbance of rapid eye movement sleep in spinocerebellar ataxia type 2. Mov Disord 2006;10:1751-1754.

9. Tuin I, Voss U, Kang JS, et al. Stages of sleep athology in spinocerbellar ataxia type 2(SCA2). Neurology 2006;67:1966-1972

10. Jardim LB, Pereira ML, Silveira I, Ferro A, Sequeiros J, Giugliani R. Neurologic findings in Machado-Joseph disease: relation with disease duration, subtypes, and (CAG)n. Arch Neurol 2001;58:899-904. 
11. Rüb U, Brunt ER, Deller T. New isights into the pathoanatomy of spinocerebellar ataxia type 3 (Machado Joseph disease). Curr Opin Neurol 2008; 21:111-116.

12. D'Abreu A, França M Jr, Conz L, et al. Sleep symptoms and their clinical correlates in Machado-Joseph disease. Acta Neurol Scand 2009;119: 277-280.

13. Schols L, Bauer P, Schmidt T, Schulte T, Riess O. Autosomal dominant cere bellar ataxias: clinical features, genetics, and pathogenesis. Lancet Neurol 2004;3:291-294.

14. Friedman JH, Fernandez HH, Sudarsky LR. REM behavior disorder and excessive daytime somnolence in Machado-Joseph Disease (SCA-3). Mov Disord 2003;18:1520-1522

15. D'Abreu A, França Jr MC, Paulson HL, Lopes-Cendes I. Caring for MachadoJoseph disease: current understanding and how to help patients. Parkinsonism Relat Disord 2010;16:2-7.

16. Boesch SM, Frauscher B, Brandauer E, et al. Restless legs syndromeand motor activity during sleep in spinocerebellar ataxia type 6. Sleep Med 2006:7:529-532.

17. Embiraçu EK, Martyn ML, Schlesinger D, Kok F. Autossomal recessive ataxias: 20 types, and counting. Arq Neuropsiquiatr 2009;67:1143-1156.

18. Brancati F, Dallapiccola B, Valente EM. Joubert syndrome and related disorders. Orphanet J Rare Dis 2010;8:5:20.

19. Boltshauser E, Herdan M, Dumermuth G, Isler W. Joubert syndrome: clinical and polygraphic observations in a further case. Neuropediatrics 1981;12:181-191.

20. Reddy PL, Grewal RP. Friedreich's ataxia: a clinical and genetic analysis Clin Neurol Neurosurg 2007:109:200-202.

21. McGrath-Morrow SA, Sterni L, McGinley B, et al. Polysomnographic values in adolescents with ataxia telangiectasia. Pediatr Pulmonol 2008; 43:674-679.

22. Stefanova, N, Bucke P, Duerr S, Wenning GK. Multiple system atrophy: an update. Lancet Neurol 2009;8:1172-1178.

23. Iranzo A. Sleep and breathing in multiple system atrophy. Curr Treat Options Neurol 2007:9:347-353.

24. Boeve BF, Silber MH, Ferman TJ, et al. Association of REM sleep behavior disorders and neurodegenerative disease may reflect an underlying synucleopathy. Mov Disord 2001;16:622-630.

25. Claassen DO, Josephs KA, Ahlskog JE, et al. REM sleep behavior disorder preceding other aspects of synucleinopathies by up to half a century. Neurology 2010;75:494-499.

26. Gama RL, Távora DG, Bomfim RC, et al. Sleep disturbances and brain MRL morphometry in Parkinson's disease, multiple system atrophy and progressive supranuclear palsy: a comparative study. Parkinsonism Relat Disord 2010; 16: 275-279.

27. Colosimo C, Morgante L, Antonini A, et al. Non-motor symptoms in atypical and secondary parkinsonism: the PRIAMO study. J Neurol 2010; 257:5-14.

28. Kamm C, Healy DG, Quinn NP, et al. The fragile X tremor ataxia syndrome in the differential diagnosis of multiple system atrophy: data from the EMSA study group. Brain 2005;128:1855-1860

29. Pareyson D, Fancellu R, Mariotti C, et al. Adult-onset Alexander disease: a series of eleven unrelated cases with review of the literature. Brain 2008; 131:2321-2331

30. Montagna P. Fatal familial insomnia: a model disease in sleep physiopathology. Sleep Med Rev 2005;9:339-353.

31. Will RG, Zeidler M, Stewart GE, et al. Diagnosis of new variant CreutzfeldtJakob disease. Ann Neurol 2000;47:575-582. 